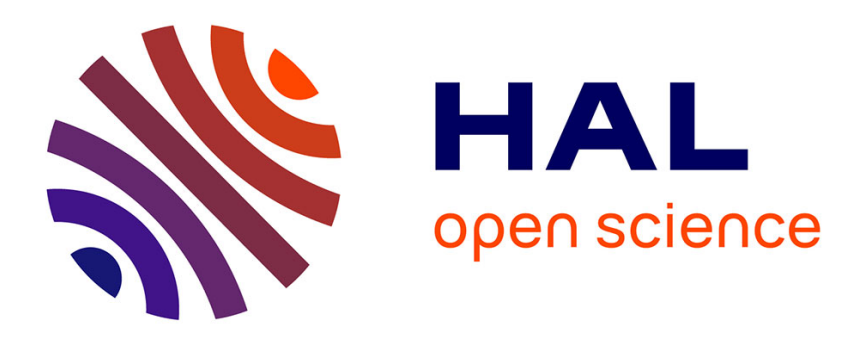

\title{
Montages culinaires, la partition du légume
}

Sylvie Leleu-Merviel

\section{To cite this version:}

Sylvie Leleu-Merviel. Montages culinaires, la partition du légume. Communication \& langages, 2010, 164, pp.53-71. hal-03186234

\section{HAL Id: hal-03186234 \\ https://hal.science/hal-03186234}

Submitted on 30 Mar 2021

HAL is a multi-disciplinary open access archive for the deposit and dissemination of scientific research documents, whether they are published or not. The documents may come from teaching and research institutions in France or abroad, or from public or private research centers.
L'archive ouverte pluridisciplinaire HAL, est destinée au dépôt et à la diffusion de documents scientifiques de niveau recherche, publiés ou non, émanant des établissements d'enseignement et de recherche français ou étrangers, des laboratoires publics ou privés. 


\section{MONTAGES CULINAIRES, LA PARTITION DU LÉGUME}

\section{Sylvie Leleu-Merviel}

NecPlus | « Communication \& langages »

$2010 / 2 \mathrm{~N}^{\circ} 164 \mid$ pages 53 à 71

ISSN 0336-1500

Article disponible en ligne à l'adresse :

https://www.cairn.info/revue-communication-et-langages1-2010-2-page-53.htm

Distribution électronique Cairn.info pour NecPlus.

(C) NecPlus. Tous droits réservés pour tous pays.

La reproduction ou représentation de cet article, notamment par photocopie, n'est autorisée que dans les limites des conditions générales d'utilisation du site ou, le cas échéant, des conditions générales de la licence souscrite par votre établissement. Toute autre reproduction ou représentation, en tout ou partie, sous quelque forme et de quelque manière que ce soit, est interdite sauf accord préalable et écrit de l'éditeur, en dehors des cas prévus par la législation en vigueur en France. Il est précisé que son stockage dans une base de données est également interdit. 


\section{Montages culinaires, la partition du légume}

\section{INTRODUCTION}

Aujourd'hui, les genres et les types de programmes télévisés consacrés au culinaire se sont beaucoup diversifiés. Outre la traditionnelle séquence " cuisine " présente dans tous les magazines depuis les débuts de la télévision, avec son incontournable figure de l'expert (de l'historique Maité à David Martin aujourd'hui), on a vu apparaître successivement : le partage social de la soirée gourmande privée doublé du challenge d'être sacré le "meilleur " de la semaine (Un dîner presque parfait), le concours de téléréalité de formation entre jeunes avec son gagnant unique (Oui chef!), programme ayant lui-même donné naissance à la figure du « jeune chef, médiateur de cuisine accessible »(Cyril Lignac), ainsi que des formes intermédiaires et/ou combinées (Un dîner presque parfait : finale nationale). La création d'une chaîne thématique dédiée, Cuisine-TV, vient encore enrichir ce paysage audiovisuel fourni.

Cet article ne prétend pas répertorier exhaustivement les divers programmes et genres repérables, ni les comparer. Il porte une attention exclusive à une forme spécifique : celle que nous appellerons « documentaire d'art " (cette dénomination sera justifiée au paragraphe 4). Il tente notamment de montrer, à partir d'un exemple, comment l'écriture filmique permet aux images et aux sons de témoigner des goûts et des saveurs.

Ainsi, le travail proposé ici se fonde sur l'analyse méthodique d'un film particulier pris en référence. Celui-ci s'intitule Alain Passard, le solfège du légume.

\section{SYLVIE LELEU-MERVIEL}

L'article propose une méthode de mise à plat des structures filmiques. Celle-ci s'appuie sur le dépouillement visuel et sonore d'un document finalisé, dépouillement qui permet d'en élaborer une partition filmique. Cet outil est mis en ouvre pour analyser le documentaire de Sophie Bensadoun, Alain Passard, le solfège du légume. II montre, à partir de cet exemple particulier, comment les ressources expressives propres à l'écriture filmique sont mobilisées pour incarner, par images et sons interposés, une représentation de la créativité artistique qu'exprime ce grand chef à travers sa cuisine.

Mots clés : médiation, langage audiovisuel, partition filmique, images, sons, liction, document 


\section{ÉLÉMENTS DE MÉDIATION EXTÉRIEURS AU FILM}

Comme l'écrit Yves Jeanneret ${ }^{1}$ à propos de l'étiquette de vin : " L'étiquette est par essence extérieure au vin. Le geste de tâter un tissu pour voir si l'étoffe en est moelleuse n'a pas d'équivalent dans l'ordre de la consommation du vin [...]. Le vin est intact avant d'être ouvert, et il se gâte dès qu'il l'est. » Dans le prolongement de cet extrait du texte, il cite d'ailleurs Raymond Dumay ${ }^{2}:$ "À moins d'être capable de humer le vin à travers le verre, il faut bien se résigner à l'étiquette, qui se trouve être le meilleur moyen de détermination connu, pour plusieurs raisons, dont la première est qu'il est le seul. »

De manière similaire, le film ne livre rien de lui-même avant visionnage, hormis les écritures qui l'accompagnent. Les écritures qui l'emballent, devrait-on dire. Avant d'analyser le film, il convient donc de prêter attention aux formes extérieures qui le médiatisent.

\section{Le titre du film}

Le titre est en quelque sorte la figure de proue des médiations extérieures. Outre qu'il nomme et permet donc de désigner, lorsqu'il est bien fait, il symbolise et signifie également beaucoup.

Rappelons-le, le film s’intitule Alain Passard, le solfege du légume.

Dès le premier abord, ce titre rend lisible la présence de deux « figures héroïques » dans ce film : tout d'abord, premier cité en ouverture, le chef Alain Passard, trois étoiles au Guide Michelin ; en clôture du titre, le deuxième héros de cette aventure, le légume.

Entre ces deux héros apparaît, subtilement codé dans le titre, le troisième « $\operatorname{actant}^{3}$ » majeur de l'histoire, un lieu : L'Arpège, le restaurant parisien d'Alain Passard. Le codage intervient bien entendu sous forme de jeu de mots et transposition terminologique de L'Arpège au « solfège ».

\section{Les indications normées du film}

Continuons à filer la métaphore de l'étiquette de vin. Yves Jeanneret poursuit ${ }^{4}$ : «Il est patent que l'étiquette est fortement normée [...]. L'étiquette est à cet égard assimilable à un formulaire, dont les rubriques sont aussi codifiées que la façon de remplir les rubriques ? " Là encore, la pochette d'un DVD comporte traditionnellement un certain nombre de rubriques systématiques, répondant à des normes d'usage plus qu'à une réglementation précise. Elles concernent les données relatives à la réalisation, à la production et à la distribution du film.

Les indications portées sur la jaquette de notre film sont les suivantes : un documentaire de Sophie Bensadoun, produit en 2006 par Nord-Ouest

1. Jeanneret, Yves, 2004, "Étiquette de vin et étiquette de table. Pouvoirs de l'écriture et figures de la sociabilité ", in Boutaud, Jean-Jacques (dir.), L'imaginaire de la table. Convivialité, commensalité et communication, L'harmattan, Paris, pp. 82-83.

2. Dumay, Raymond, 1967, Guide du vin, Stock, Paris, p. 58.

3. L'actant est une notion héritée de la sémiotique de Greimas et de son modèle actantiel : Greimas, A., 1966, Sémantique structurale, Larousse, Paris.

4. Jeanneret, Yves, 2004, op. cit., p. 84. 
Documentaires avec la participation de France 5 et le soutien du CNC, du département de la Sarthe et des Régions Limousin et Pays de la Loire ; édité et diffusé en DVD depuis 2007 par La Huit Distribution.

\section{Le pitch du film}

Outre ces premiers éléments saillants de perception du film, beaucoup de supports de communication, dont la jaquette de la boîte, comportent un court texte qui n'est ni purement descriptif, ni purement commercial. Appartenant sans conteste au genre des écrits promotionnels, il répond à la fonction principale d'accroche : il s'agit donc de présenter et d'attirer, mais sans trop dévoiler. Les professionnels ont renoncé à la traduction du mot anglo-saxon unanimement employé : le pitch.

Le pitch proposé pour le film est le suivant :

Entré en cuisine à 14 ans comme d'autres entreraient en religion, Alain Passard fait ses classes auprès des plus grands avant de créer son propre restaurant L'Arpège, à Paris, auréolé depuis plus de 10 ans de 3 étoiles au Guide Michelin. Un parcours d'autant plus exemplaire que l'homme a bousculé le petit monde conservateur de la haute gastronomie : ce qui était au bord de l'assiette, il l'a mis au centre, et il a supprimé la viande rouge qui lui avait pourtant donné sa réputation de maître de la cuisson, pour faire du légume le roi de l'assiette. Pour accomplir ce changement radical, Alain Passard a créé son propre potager à Fillé-sur-Sarthe. Pas moins de 500 variétés de fruits et légumes y sont cultivées dans le plus grand savoir-faire. Ce film l'a suivi pendant plusieurs mois et célèbre ce chef d'orchestre atypique, ainsi que ses plats harmonieusement colorés, dignes des plus grands tableaux.

L'impression « induite » par le titre d'un récit doublement héroïque, dont les deux figures sont Alain Passard et le légume, est donc totalement confirmée par la deuxième phrase du pitch. Outre le premier lieu d'ancrage du film codé dans le titre, le restaurant L'Arpège, le pitch met en avant dans sa troisième phrase un second lieu décisif, le potager créé par le chef à Fillé-sur-Sarthe (ce qui explique la mobilisation du département de la Sarthe et des deux Régions pour le financement du film). On sent donc poindre ici le « fil rouge ${ }^{5}$ » qui conduira de la culture du légume dans le potager de Fillé-sur-Sarthe à l'assiette servie à L'Arpège en passant par la préparation et la cuisson au piano de sa cuisine, chaîne de valeur sur laquelle Alain Passard règne en maître puisqu'il en contrôle toutes les étapes.

\section{Éléments structurants du film}

Les outils de technique d'écriture audiovisuelle assoient la conception d'un film sur la détermination de trois éléments structurants :

- la forme de traitement et l'espace de liberté associé ;

- la ligne directrice et le fil rouge ;

- l'unité thématique.

La forme de traitement est ici celle du documentaire. Elle implique de ne recourir à aucune forme de «fabrication fictionnelle». Le documentariste enregistre à l'image et au son des moments de vie sur lesquels il n'intervient pas, autrement que par

5. Le fil rouge désigne l'élément de continuité narrative autour duquel se tisse un documentaire. 
sa présence et celle de son équipe qui, fatalement, modifient les comportements. Un documentariste professionnel ne demande jamais à un personnage de ressortir et de rouvrir une porte parce qu'il doit refaire le plan. S'il n'a pas le plan, il s'en passe. Par conséquent, l'événement, les faits réels de l'instant vécu dans toute son authenticité priment. La caractéristique principale du documentaire est donc que l'espace de liberté par rapport au réel est réduit à néant. Cela n'implique pas l'absence d'écriture. Au contraire, la suite montrera que si l'espace de liberté du documentariste ne porte pas sur les événements captés, il est intégralement transféré sur l'écriture qui, elle, est très élaborée. Décider de filmer ça, et pas ça, et de le tourner de telle manière et pas de telle autre, constitue quoi qu'il en soit le cœur de la décision au tournage.

La ligne directrice est la force qui conduit la narration depuis le départ jusqu'à son aboutissement. Elle se construit en structurant des liens qui vont courir tout au long du sujet. Ces liens peuvent être de plusieurs natures : personnages, lieux, actions, objets... le tissage de ces divers fils rouges compose la ligne directrice. Le pitch a permis d'identifier ces divers éléments et de les recenser au paragraphe précédent.

Il ne manque donc que l'unité thématique pour parachever la construction abstraite. L'unité thématique englobe bien sûr le thème principalement traité (sans exclusive de sous-thèmes secondaires). Souvent, celui-ci s'exprime au final par un seul mot : la solitude, la maladie, la chance, la vengeance... Mais ce simple mot ne suffit pas. L'unité thématique exprime plus précisément la clef, l'âme du problème, la dimension et la concrétisation données à ce thème. Dans le registre dramatique, Patrice Pavis propose un modèle pour l'analyse du texte dramatique qu'il avoue inspiré de Lector in fabula d'Umberto $\mathrm{Eco}^{6}$. Dans ce modèle, le dernier niveau proposé est celui du sens, qu'il définit comme ${ }^{7}$ : «la question idéologique de la thèse [...]. Cette case est la boîte noire du non-dit. » Quoique se rapportant à un autre registre d'écriture, cette définition circonscrit assez justement ce qu'est l'unité thématique dans la construction filmique.

Puisque l'unité thématique relève de l'ordre du non-dit, on ne peut espérer la trouver explicitement exprimée dans les documents médiateurs du film. Néanmoins, on peut relever à nouveau la métaphore musicale, du piano du cuisinier à L'Arpège qu'il compose, métaphore reprise par le "solfège » du titre. La dernière phrase du pitch, après avoir renforcé ce premier axe avec la notion de " chef d'orchestre ", laisse poindre un nouveau registre : celui de l'harmonie colorée et du tableau pictural.

\section{Bilan}

On constate que, avant d'avoir vu la moindre image de ce film, beaucoup de choses sont déjà dévoilées à qui sait lire et interpréter les documents médiateurs. On remarque surtout une rigueur de construction qui transparait bien avant d'avoir

6. Eco, Umberto, 1985, Lector in fabula. Le rôle du lecteur ou la coopération interprétative dans les textes narratifs, Grasset, Paris.

7. Pavis, Patrice, 2002, Le théâtre contemporain. Analyse des textes de Sarraute à Vinaver, Nathan, Paris, p. 22. 
accédé au film. Ceux qui pensaient que faire un film est à la portée de tous en sont d'ores et déjà pour leurs frais.

On aurait pu effectuer une analyse de discours pour faire émerger, du registre syntaxique mobilisé, un ensemble d'items relatifs à l'excellence (les plus grands, auréolé, parcours exemplaire, réputation de maître, le plus grand savoir-faire...). On aurait pu également décortiquer le visuel de la jaquette et l'iconographie associée.

Néanmoins, notre propos n'étant pas d'analyser la médiation du film, mais bien la médiation par le film, il est temps de passer des amuse-bouches au plat principal.

\section{Partition du film}

Le premier problème que l'on rencontre, dans cet exercice d'exploration sémiotique d'un film, est de disposer d'un modèle formel permettant de représenter le film afin d'en supporter l'analyse. En effet, la fiction dispose d'un outil de représentation textuelle - le scénario - et d'un outil de représentation graphique - le story-board. Cependant, ni l'un ni l'autre ne sont adaptés à la forme documentaire.

Pour s'être penchée sur une problématique avoisinante concernant les séries télévisées, Carmen Compte met en œuvre ce qu'elle désigne par " une méthode de mise à plat des structures narratives ${ }^{8}$. »C C'est dans un esprit similaire et dans le prolongement de ses travaux que vont être forgés les outils qui nous sont nécessaires.

\section{Principe de mise à plat de la structure filmique}

Carmen Compte indique en effet : "L'analyse commence par une mise à plat du document, car celle-ci sert de cadre de référence. Il s'agit de mettre sous forme de tableau les éléments tels qu'ils ont été perçus. Elle donne à voir la chronologie des événements, à saisir rapidement le découpage des unités constitutives. Leur minutage donne en outre une information importante sur les éléments mis en avant par le réalisateur. Il ne s'agit cependant pas d'un script précis et rigoureux ou d'un scénarimage (story-board). »

Plusieurs éléments usuels interviennent dans la mise à plat qu'elle propose :

- des unités d'action ou événementielles, considérées comme des séquences et repérées par leur numéro de séquence ;

- le TC-In (time-code d'entrée) et TC-Out (time-code de sortie), employés par le monteur comme repérage de la séquence dans la continuité temporelle du film;

- la différence entre TC-In et le TC-Out fournit la durée de la séquence ;

- des indications scripto-visuelles, formes d'écritures descriptives, codées ou en langage naturel, de ce qu'on voit à l'image ;

- des indications scripto-sonores, formes d'écritures descriptives, codées ou en langage naturel, de ce qu'on entend au son...

8. Compte, Carmen, 2009, "L'influence des soap opera sur les stratégies narratives des séries télévisées », RIHM, vol. $9, \mathrm{n}^{\circ} 2$, p. 8 . 
Notons que ces éléments sont similaires à ceux que note la scripte dans les deux documents qu'elle a charge de produire, outre le rapport journalier, à chaque jour de tournage : respectivement le rapport image et le rapport son. Avec tout de même une différence notoire : les notations de la scripte portent sur les rushes, c'est-à-dire la matière brute issue de la captation, et pas du tout sur le résultat aujourd'hui extrêmement transformé que représente un film après l'étape de postproduction, qui fait l'objet du présent travail.

Ce sont également, peu ou prou, les éléments que comporte une feuille de dérushage, qui est l'opération préliminaire au montage. Cependant, la même remarque s'applique. Comme son nom l'indique, le dérushage est un travail qui porte sur les rushes, et non sur un document finalisé, après montage et postproduction.

Au demeurant, il s'avère que non seulement l'outil nous manque, mais aussi un nom pour désigner ce qu'il va produire. Carmen Compte condamne le vocable de "script ", sans doute à juste titre car il engendre beaucoup de confusion et ne désigne pas une réalité bien établie (en France tout du moins, puisque c'est en revanche le terme adéquat pour désigner le «scénario » aux États-Unis). Les termes de « rapports " qu'emploie la scripte ne sont guère plus convaincants.

Poursuivant l'analogie avec le monde musical que suggère notre documentaire lui-même, cet article promeut finalement la notion de "partition ", dont le sens littéral traduit assez fidèlement la mise en évidence du découpage en unités constitutives. D'autre part, au même titre que la partition musicale fait connaître tout de la musique, sauf la musique, la partition filmique révèle tout du film, sauf la matière filmique. L'appellation est donc conceptuellement juste. Quoi qu'il en soit, elle est adoptée pour la suite de l'article.

\section{Partition macro-structurelle}

Le fait de travailler à partir d'une édition DVD présente un avantage majeur que ne comporte pas le film : le réalisateur y a déjà effectué un chapitrage, c'est-à-dire un premier découpage en unités constitutives qu'il juge cohérentes. L'intérêt est bien entendu de lever toute forme d'ambiguïté. Puisque le découpage en chapitres a été effectué par le réalisateur lui-même, il est exempt de toute interprétation de la part de l'analyste.

Il s'agit d'une première partition de granularité importante : on peut la comparer au découpage en numéros d'une pièce musicale. Notre documentaire, de 52 minutes conformément au format français en usage pour les " grands documentaires ", est ainsi découpé en 11 chapitres. La partition correspondant à ce découpage macro-structurel est proposée en figure 1.

De la même manière que les numéros de la partition musicale portent parfois des titres (pour exemple, Les Choéphores, pièce de Darius Milhaud sur un texte de Paul Claudel, est ainsi composée successivement de $: \mathrm{n}^{\circ} 1$ "Vocifération funèbre ",

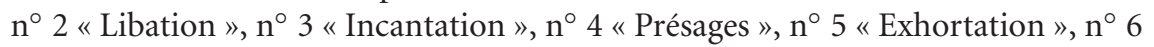
"La justice et la lumière ", $\mathrm{n}^{\circ} 7$ «Conclusion »), un titre a été porté en dernière colonne de la partition macro-structurelle. Il indique la dominante et/ou le thème du chapitre correspondant. 


\begin{tabular}{|c|c|c|c|c|}
\hline $\begin{array}{l}\text { Chapitre } \\
\mathbf{N}^{\circ}\end{array}$ & TC-In & TC-Out & Durée & Dominante/" thème " \\
\hline 1 & $00: 00$ & $05: 15$ & $5^{\prime} 15^{\prime \prime}$ & $\begin{array}{l}\text { Ouverture, arrivée à L'Arpège } \\
\text { "La gastronomie du légume " }\end{array}$ \\
\hline 2 & $05: 15$ & $08: 36$ & $3^{\prime} 21^{\prime \prime}$ & $\begin{array}{l}\text { Interview d'Alain Passard } \\
\text { "Le renouveau créatif " }\end{array}$ \\
\hline 3 & $08: 36$ & $14: 29$ & $5^{\prime} 53^{\prime \prime}$ & $\begin{array}{l}\text { Potager à Fillé-sur-Sarthe } \\
\text { "La créativité du légume " }\end{array}$ \\
\hline 4 & $14: 29$ & $20: 53$ & 6' 24" & $\begin{array}{l}\text { Lycée hôtelier à Granville } \\
\text { "Le raffinement du geste " }\end{array}$ \\
\hline 5 & $20: 53$ & $24: 00$ & 3'07" & $\begin{array}{l}\text { Retour à L'Arpège } \\
\text { "Le choix du vin " }\end{array}$ \\
\hline 6 & $24: 00$ & $27: 00$ & $3{ }^{\prime} 00^{\prime \prime}$ & $\begin{array}{l}\text { Dîner de gala à Tokyo } \\
\text { "Instabilité et mise en danger " }\end{array}$ \\
\hline 7 & $27: 00$ & $30: 16$ & $3^{\prime} 16^{\prime \prime}$ & $\begin{array}{l}\text { Le rôtisseur de L'Arpège } \\
\text { "Les sons du piano et la main qui } \\
\text { joue " }\end{array}$ \\
\hline 8 & $30: 16$ & $37: 17$ & 7'01" & $\begin{array}{l}\text { Les cuissons à L'Arpège } \\
\text { "Tableau et peinture, dans le } \\
\text { légume " }\end{array}$ \\
\hline 9 & $37: 17$ & $44: 15$ & $6^{\prime} 58^{\prime \prime}$ & $\begin{array}{l}\text { Potager à Fillé-sur-Sarthe } \\
\text { "Nouveau légume, nouvelles } \\
\text { cuissons " }\end{array}$ \\
\hline 10 & $44: 15$ & $51: 04$ & 6' 49" & $\begin{array}{l}\text { Le grand chef de L'Arpège } \\
\text { "Donner une saveur et un parfum à } \\
\text { une couleur" }\end{array}$ \\
\hline 11 & $51: 04$ & $52: 16$ & 1' 12" & $\begin{array}{l}\text { Coda } \\
\text { "Arpèges virtuoses" }\end{array}$ \\
\hline
\end{tabular}

Figure 1 : Partition macro-structurelle, découpage en chapitres

On pourra remarquer que, comme souvent dans le texte dramatique et même dans le roman de facture classique, un changement de chrono-tope (lieu et/ou temps) induit un changement d'unité structurelle. On observe ainsi une structuration parfaitement rythmée du documentaire :

- $\mathrm{n}^{\circ} 1$ arrivée à L'Arpège,

- $\mathrm{n}^{\circ} 2$ séquence interview d'Alain Passard,

- $\mathrm{n}^{\circ} 3$ potager de la Sarthe, 
- $\mathrm{n}^{\circ} 4$ lycée hôtelier à Granville,

- $\mathrm{n}^{\circ} 5$ retour à L'Arpège,

- $n^{\circ} 6$ soirée de gala à Tokyo ;

puis de nouveau, à la fin :

- $\mathrm{n}^{\circ} 9$ potager de Fillé-sur-Sarthe,

- $\mathrm{n}^{\circ} 10$ retour à L'Arpège,

- $\mathrm{n}^{\circ} 11$ coda.

On observe, en revanche, une perte de dynamique rythmique sur les séquences 7 et 8 , moins carrées du point de vue de la construction, qui mélange des extraits de l'interview avec des plans de préparation en cuisine. Ce sont les 10 minutes de « tunnel» bien connues du documentariste : elles se situent presque toujours entre 30 et 40 minutes dans la forme longue du 52 minutes, qui impose de reprendre son souffle pour tenir la distance avant d'attaquer le sprint final.

\section{Partition micro-structurelle de l'ouverture - chapitre 1}

Quand on dispose du découpage en numéros d'une partition, on n'a toujours pas la musique. De la même manière, il s'agit désormais d'élaborer la partition filmique de chaque chapitre avec une granularité et un niveau de détail bien plus fins. Carmen Compte indique ainsi : «Une analyse sémiotique plus poussée exige de détailler les séquences en plans selon leur grosseur et leur durée et de repérer où se situent les effets spéciaux apportés par un mouvement particulier de la caméra (panoramique, travelling) ou de l'objectif $(z o o m)^{9} »$. Le niveau de détail auquel elle fait référence pour l'image doit bien entendu trouver son équivalent pour la composante sonore, qui fait l'objet d'un travail de mixage également très élaboré où sont associés et dosés les voix, les sons d'ambiance, les bruitages, la musique, etc

Tout auteur connaît l'importance d'un moment phare qu'il convient de soigner tout particulièrement : le début, l'incipit littéraire, l'ouverture musicale, qui jouent le rôle d'accroche. Le discours filmique ne déroge pas à cette règle générale. C'est pourquoi nous allons illustrer la mise en œuvre de la partition micro-structurelle sur ce «moment choisi ».

Ainsi, la partition correspondant au découpage micro-structurel des 42 premières secondes du chapitre 1 est proposée en figure 2.

Compte tenu de la durée de 42 secondes pour une séquence initiale de 5 minutes 15 secondes, on peut considérer ce fragment filmique comme l'exact équivalent de l'incipit, à savoir la toute première phrase du discours. En effet, la fin de ces 5 premières unités constitutives marque l'entrée dans une toute autre phase (resp. phrase).

Plusieurs constatations s'imposent au vu de cet exemple de partition micro-structurelle. D'une part, la quantité d'éléments à faire figurer plaide pour une édition sur format A3. Carmen Compte indique d'ailleurs que, pour le 


\begin{tabular}{|c|c|c|c|c|c|}
\hline $\mathrm{N}^{\circ}$ & TC-In & TC-Out & Durée & Rapport Image & Rapport Son \\
\hline 1 & $00: 00$ & $00: 06$ & $6 "$ & $\begin{array}{l}\text { Sillons dans la terre, PS } \\
\text { Incrustation générique : } \\
\text { " Nord-Ouest } \\
\text { Documentaires avec la } \\
\text { participation de } \\
\text { France } 5 \text { " }\end{array}$ & $\begin{array}{l}\text { Musique : air assez } \\
\text { "solfégique " } \\
\text { au piano } \\
\text { Bruits de hachage }\end{array}$ \\
\hline 2 & $00: 06$ & $00: 12$ & $6 "$ & $\begin{array}{l}\text { Chou argenté, GP } \\
\text { Incrustation générique: } \\
\text { "Un film } \\
\text { de Sophie Bensadoun " }\end{array}$ & Idem \\
\hline 3 & $00: 12$ & $00: 16$ & $4 "$ & $\begin{array}{l}\text { Légume cuit dressé pour } \\
\text { service, TGP } \\
\text { Incrustation générique: } \\
\text { "Le solfège du légume " }\end{array}$ & Idem \\
\hline 4 & $00: 16$ & $00: 31$ & $15 "$ & $\begin{array}{l}\text { TVG Passard marchant } \\
\text { dans la rue, ouverture } \\
\text { porte extérieure } \\
\text { Plan dans le mouvement } \\
\text { Incrustation : "Alain } \\
\text { Passard, chef } 3 \text { étoiles } \\
\text { de L'Arpège " }\end{array}$ & $\begin{array}{l}\text { Idem + ambiance rue } \\
\text { + voix off } \\
\text { "Un jour, cet homme } \\
\text { annonce que le } \\
\text { légume, toujours } \\
\text { posé sur le bord de } \\
\text { l'assiette, il va le } \\
\text { mettre au centre et } \\
\text { en faire le plat } \\
\text { principal. Un gratin } \\
\text { d'oignons à la place } \\
\text { d'une côte de bœuf : } \\
\text { de quoi faire trembler } \\
\text { les } 3 \text { étoiles de son } \\
\text { restaurant parisien } \\
\text { L'Arpège. " }\end{array}$ \\
\hline 5 & $00: 31$ & $00: 42$ & $11 "$ & $\begin{array}{l}\text { Raccord Passard } \\
\text { ouvrant la porte et } \\
\text { marchant dans l'entrée } \\
\text { intérieur } \\
\text { L'Arpège, fermeture } \\
\text { portes battantes cuisine } \\
\text { Plan dans le mouvement } \\
\text { Incrustation : "L'Arpège, } \\
\text { Paris " }\end{array}$ & $\begin{array}{l}\text { Idem + ambiance } \\
\text { intérieur (bonjour } \\
\text { chef; bonjour } \\
\text { Sandra) + voix off } \\
\text { " Mais Alain Passard } \\
\text { les a gardées, et } \\
\text { continue chaque jour } \\
\text { d'inventer la } \\
\text { gastronomie du } \\
\text { légume." }\end{array}$ \\
\hline
\end{tabular}

$\mathrm{PS}=$ plan serré, $\mathrm{GP}=$ gros plan, $\mathrm{TGP}=$ très gros plan, $\mathrm{TVG}=$ travelling .

Figure 2: Partition micro-structurelle du chapitre 1 « ouverture " 
dépouillement de séries complètes, elle a effectué un reporting séparé en trois documents complémentaires : un rapport image, un rapport son, et un rapport technique - éléments qui sont tous présents en même temps dans l'exemple ci-dessus. En effet, si l'on rapporte les 42 secondes ci-dessus aux 52 minutes du documentaire, on voit que ce sont des pages et des pages que nécessite l'édition de la partition complète.

Mais surtout, ce début suffit amplement à mesurer la qualité de l'écriture filmique de ce documentaire. En effet, sur 5 plans image, l'essentiel est dit. Sous le générique, il ne faut que 3 plans fixes raccordés en cut et 16 secondes pour passer du sillon dans la terre au légume à maturité, puis au légume cuit dressé pour le service, traduction visuelle « littérale » du fil rouge du document. Le héros principal, Alain Passard, entre en scène au quatrième plan et à la seizième seconde. Entrée à L'Arpège (où l'on est accueilli par un bonjour), au cinquième plan et à la trentième seconde. Alain Passard est à l'image pendant 25 secondes sur les 42 (soit $60 \%$ du temps), il y est toujours en marche (homme qui bouge, homme d'action), il y est aimable (il dit bonjour) et souriant. Le légume, lui, est présent à l'image 16 secondes sur les 42 (soit $38 \%$ du temps) - si l'on admet que le sillon est un légume en germination.

La qualité du travail sonore n'est pas en reste. Le premier son entendu est le bruit du hachage d'un légume. Il est rapidement accompagné d'un air plutôt scolaire, d'esprit assez "solfégique ", bien entendu au piano. Dès la fin du générique, la partition sonore devient complexe, puisqu'elle mixe musique, bruitages, sons d'ambiance et voix off. Notons que ce sera la seule présence de la voix off dans tout le documentaire. On peut dès lors la considérer comme le «Monsieur Loyal » du document, ou dans un registre plus télévisuel, l'animateur qui présente la retransmission d'un concert en quelques mots.

Jusqu'au commentaire délivré par la voix off ou l'incrustation textuelle présentant Alain Passard, tous ces éléments sont parfaitement congruents avec ce qui avait émergé de l'analyse menée au paragraphe 2 : l'écriture filmique est en adéquation stricte avec les écritures de médiation qui accompagnent le document, ce qui témoigne d'un travail considérable de construction théorique. Cette analyse rapide (que l'on pourrait compléter jusqu'au moindre détail : synchronisation des images avec la phrase musicale, raccords colorimétriques, etc.) montre qu'il s'agit bien d'une ouverture au sens musical du terme, et justifie a posteriori le titre que nous avions donné à cette séquence dans la partition macro-structurelle. Elle remplit à merveille sa fonction : annoncer au spectateur la teneur et la substance des 51 minutes qui vont suivre.

\section{Bilan}

Bien entendu, la place manque pour faire figurer ici l'intégralité des partitions de ce film. Néanmoins, ce paragraphe a exposé le principe du dépouillement et en a proposé des exemples de mise en ouvre, qui permettent de s'approprier ces outils au gré des besoins. L'intérêt de la méthode de dépouillement qui conduit à l'élaboration de la partition est qu'elle ne laisse aucune place à l'interprétation subjective : la partition est un outil objectif, qui décrit rigoureusement les unités 
visuelles et sonores composant le document. Celle-ci sert de base à l'analyse qui est effectuée dans un deuxième temps.

Les premiers éléments d'analyse mettent en évidence la rigueur de la construction audiovisuelle, dans toute la complexité de la dimension langagière qui lui est propre. En effet, c'est sa spécificité de marier des images fixes, des images en mouvement, du langage verbal, des bruits d'ambiance, des bruitages, de la musique, le tout associé via des raccords et des manipulations techniques élaborés.

Néanmoins, une question fondamentale n'a pas encore trouvé réponse : comment un langage adressé à l'œil et à l'oreille permet-il de rendre compte d'une composition gustative?

\section{MÉdiation gourmande}

Autant la forme documentaire semble pertinente pour nous toucher par la contemplation des grands paysages naturels, autant la musique se prête à un traitement audiovisuel, autant il paraît bien incertain de pouvoir traiter de saveurs à l'aide d'images et de sons.

C’est là une vraie question qui mérite une attention un peu soutenue.

\section{Réponse des sciences cognitives}

Le petit opus de Françoise Léon dans la collection des «Pommes du Savoir » fournit quelques éléments qui contribuent à la compréhension du phénomène. Elle ouvre son ouvrage par ce récit : «En passant devant la boulangerie, mon attention fut captée par la délicieuse odeur qui s'en échappait. Je me suis alors souvenue qu'il fallait que j'achète du pain. La baguette que l'on me proposa était encore tiède, je ne résistai pas à l'envie d'en détacher le quignon. La croûte était dorée et craquante, la mie encore chaude était blanche et aérée, l'odeur de pain frais me chatouillait les narines. Je croquai à pleines dents dans le quignon, ce bon goût de pain grillé et l'arôme de cette mie tendre, légèrement salée, me donnèrent du plaisir, je savourai cet instant. . ${ }^{10} »$ La psychologue cognitiviste montre ensuite que l'odeur véhiculée par les molécules volatiles odorantes, la vue de l'aliment et notamment sa couleur, la tiédeur perçue grâce au contact du pain avec la peau, le son du craquant de la croûte attestent que les quatre sens de l'olfaction, de la vision, et dans une moindre mesure de l'audition et du toucher, entrent en relation, se conjuguent et interviennent bien avant la mise en bouche de la nourriture, qui déclenche le goût, seul sens à n'intervenir qu'après l'ingestion. Bien plus, l'olfaction et la vision, principalement, permettent à l'individu de se faire une représentation de ce qui va être mangé, préparent l'organisme à l'accueil de l'aliment et anticipent la prise alimentaire (la montée de l'eau à la bouche en étant l'un des indicateurs perceptibles). 
Outre la dimension sensorielle liée à la perception de l'aliment par les cinq sens, le plaisir ou le dégoût alimentaires revêtent au surplus :

- une dimension cognitive, liée à la représentation que l'on a des aliments. Les fonctions du langage, de la mémoire et de la pensée y sont impliquées ;

- une dimension causale, associative, liée aux conséquences bénéfiques ou nocives de l'ingestion.

Enfin, l'émotion participe pleinement du processus. Comme le note Françoise Léon : «L'aliment peut susciter une émotion forte par le biais d'un souvenir ou d'une représentation [...]. En décrivant l'aliment - son apparence, son odeur, son goût, sa texture, le bruit qu'il fait -, ce qu'il évoque, ce que nous savons de lui, sa richesse nous apparaît et nous nourrit déjà, tous nos sens sont mis en alerte. ${ }^{11}$ »

\section{Langage audiovisuel et goût}

La présentation des protocoles expérimentaux ayant permis de valider la pertinence scientifique des observations ci-dessus n'a bien entendu pas sa place ici. Néanmoins, il est important d'intégrer qu'il ne s'agit pas de vagues conjectures soutenues par la seule intuition, mais d'hypothèses ayant donné lieu à une vérification par la preuve. C'est un fait attesté par les travaux de sciences cognitives : une simple évocation provoque en nous, par association, des réactions en chaîne qui mobilisent tous nos sens et vont jusqu'à susciter les mêmes réactions que la présence effective et la préparation physiologique à l'ingestion.

Nous avons introduit ailleurs ${ }^{12}$ le terme de "liction » pour désigner ce phénomène de reliances complexes où le tissage de sollicitations perceptuelles, dans un contexte donné, déclenche des associations et de multiples relations où entrent en jeu des représentations antérieures, du vécu, de l'émotion, des lambeaux de passé et leur mémoire gravée en nous, le tout produisant des émergences novatrices totalement absentes des données sensorielles d'origine.

Par l'intermédiaire de Françoise Léon, le résultat auquel nous parvenons ici est que l'image d'un plat, notamment ses couleurs, mais aussi les formes et les reliefs évocateurs de textures, et dans une moindre mesure le bruit de la préparation et de la cuisson, suffisent à évoquer en nous la saveur, l'arôme et le toucher qui nous font cruellement défaut dans la représentation audiovisuelle. Ceci suffit à attester la capacité de la représentation audiovisuelle à traduire le gustatif.

\section{Mise en œuvre dans " Le solfège du légume "}

Reprenons les trois premiers plans du documentaire tels qu'ils ont été consignés dans la partition de la figure 2. Ils présentent un parfait exemple de liction audiovisuelle. En effet, les données objectives sont constituées de 3 plans fixes sans lien apparent : un sillon dans la terre, un splendide légume argenté, un dressage sur assiette d'une préparation magnifiquement dorée. Placé face à cette succession

\section{Ibid.}

12. Leleu-Merviel, Sylvie, 2008, « Du sens comme une attraction lictionnelle entre fragments rhapsodiques ", in Schauder, Silke (dir.), Camille Claudel. De la vie à l'ouvre, regards croisés, L'Harmattan, Paris, pp. 139-152. 
de plans, notre cerveau invente un lien (qui n'existe pas dans les images). Notre culture vient alors à la rescousse pour nous faire valoir que le légume du deuxième plan pousse dans la terre du premier, et que le plat du troisième plan nécessite un légume cousin germain de celui du deuxième. Nous inférons donc, par liction, cette relation logique qui fait passer du sillon dans la terre, au légume à maturité, puis au légume cuit dressé pour le service. Encore une fois, ce lien logique n'existe pas dans les images : il est simplement suggéré par la concaténation temporelle des plans. Tout individu qui méconnaîtrait nos modes de culture ou s'alimenterait de gélules serait dans l'incapacité d'établir ce lien logique. On voit donc à quel point la liction est contextuelle, et culturellement dépendante. De même, toute personne qui n'aurait jamais mangé de légumes, ou qui n'aurait jamais eu de plaisir à la dégustation de légumes, serait émotionnellement plus démunie, et par voie de conséquence forcément moins touchée par le visionnage de ce film (voire dégoûtée si elle fait une allergie aux légumes).

Rappelons que ces premiers plans sont accompagnés par un bruit de hachage, ce qui augmente le potentiel lictionnel de la séquence, en mobilisant la présence de deux sens au lieu d'un pour en susciter trois autres au lieu de quatre.

Bien entendu, le documentaire fait largement appel à des images dont la qualité esthétique favorise le phénomène d'évocation gustative explicité au paragraphe précédent.

\section{Bilan}

Ce paragraphe a montré que les évocations visuelle et olfactive, et dans une moindre mesure, sonore et tactile, appellent, anticipent et préparent le goût, et se tissent avec lui. Cela suffit à qualifier l'audiovisuel pour représenter le gourmand.

Est-ce la seule raison pour laquelle ce documentaire fonctionne si parfaitement ? Le dernier paragraphe va montrer qu'Alain Passard lui-même y apporte une touche prépondérante.

\section{Alain Passard, artiste lictionnel virtuose du légume}

Bien entendu, la forme du documentaire accorde une large place à la verbalisation, à travers les interviews et la restitution des dialogues entre les personnes présentes dans les différentes séquences. À ce titre, la parole d'Alain Passard occupe une proportion très importante des 52 minutes de la partition sonore.

Le dépouillement de la partition verbale montre alors que, dans l'exacte continuité de l'analyse cognitive menée au paragraphe précédent, Alain Passard crée davantage avec les quatre autres sens qu'avec le goût.

\section{Le visuel}

Dans cette partie, nous n'allons pas procéder à l'élaboration d'une partition exhaustive en continuité, mais concaténer des extraits de partition centrés sur la parole d'Alain Passard (le rapport image est donc ici tout à fait secondaire).

Les fragments présentés dans le tableau de la figure 3 attestent qu'Alain Passard crée ses plats avec le regard avant toute chose.

Alain Passard le dit lui-même, sa cuisine se compose comme un film : elle est lictionnelle. Cet homme est un être lictionnel. Dans sa création, il procède à des 


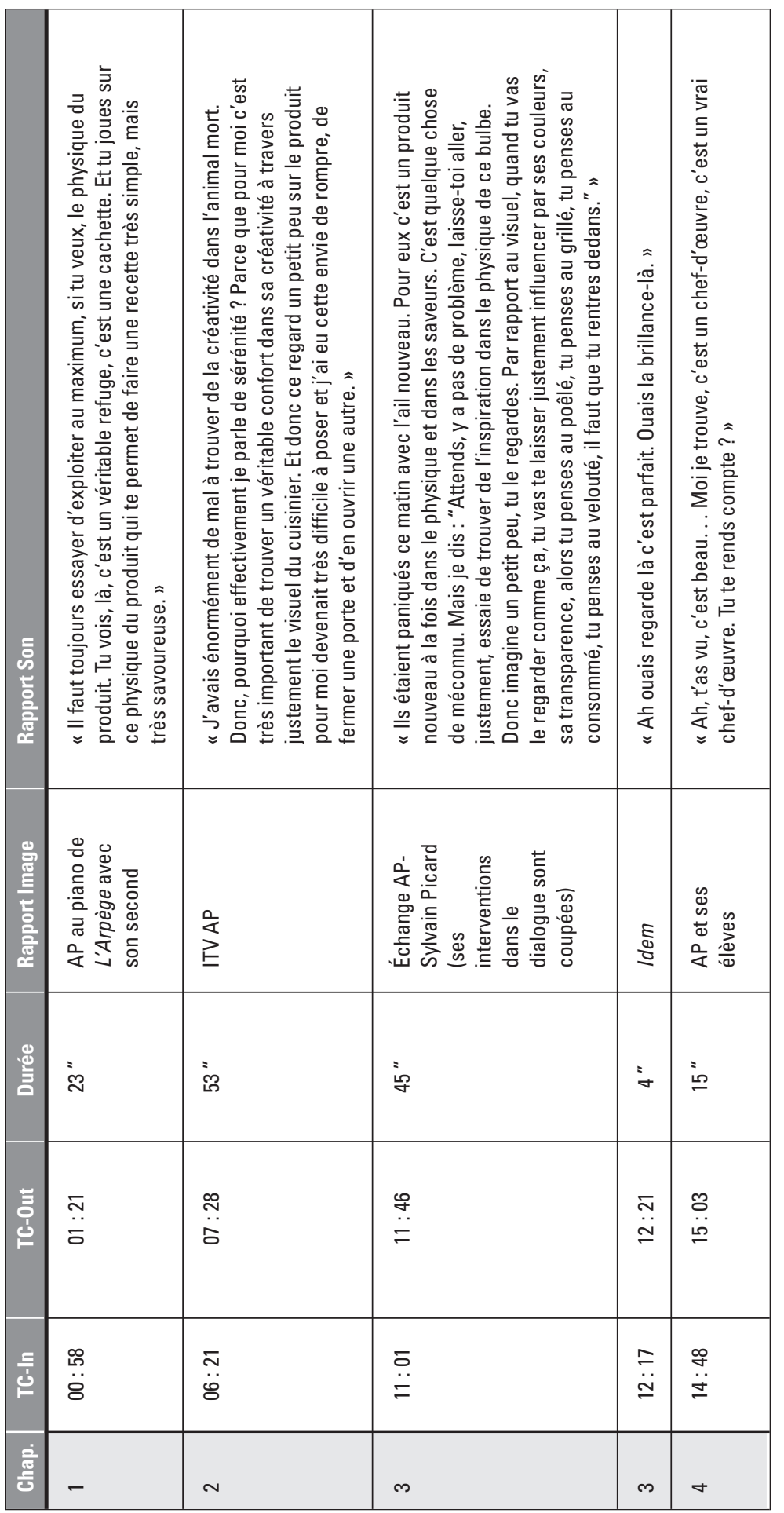




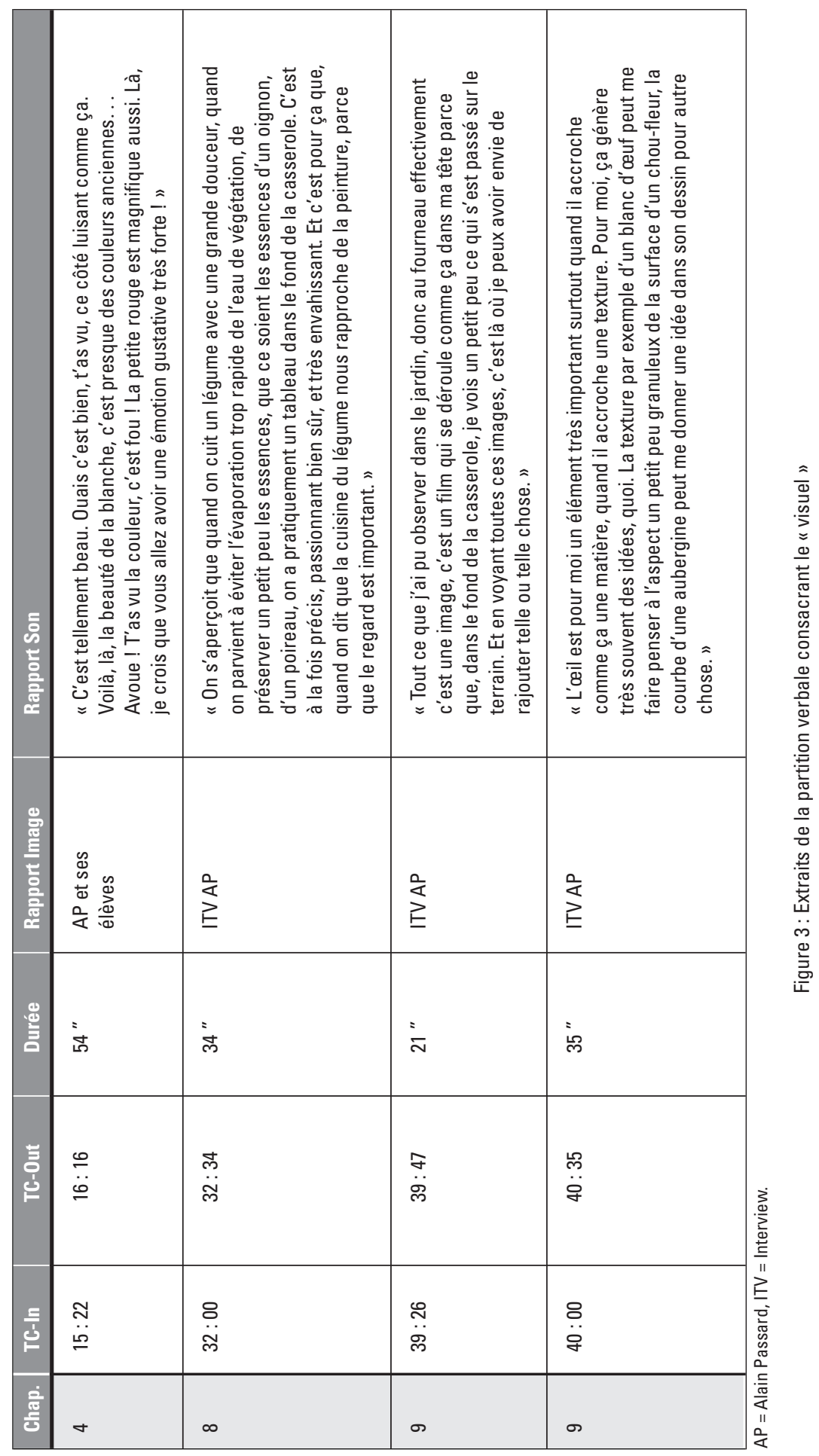


montages culinaires, exactement comme le fait, à partir d'images et de sons, le réalisateur audiovisuel dont nous avons tenté ici d'analyser le travail.

\section{Les sons}

Le nombre d'extraits contenus dans la figure 4 témoigne de l'importance que le chef octroie au visuel. L'extrait de partition ci-dessous, ici encore centré sur la parole d'Alain Passard, montre que s'il accorde la plus grande importance au visuel, les sons ne sont pas en reste.

\begin{tabular}{|c|c|c|c|c|c|}
\hline Chap. & TC-In & TC-Out & Durée & $\begin{array}{c}\text { Rapport } \\
\text { Image }\end{array}$ & Rapport Son \\
\hline 7 & $27: 46$ & $28: 32$ & $48 "$ & ITV AP & $\begin{array}{l}\text { "Le bruit en cuisine, c'est-à-dire, } \\
\text { c'est cette grand-mère qui était } \\
\text { vraiment un très grand rôtisseur qui } \\
\text { m'a appris à écouter un petit peu mes } \\
\text { cuissons parce que c'est vrai que } \\
\text { l'oreille est quelque chose pour moi } \\
\text { d'assez déterminant en cuisine. Cette } \\
\text { grand-mère avait vraiment l'oreille } \\
\text { quoi, elle savait quand il fallait } \\
\text { réguler un petit peu son four, quand il } \\
\text { fallait entrouvrir la porte du four pour } \\
\text { justement laisser un petit peu } \\
\text { s'échapper à la fois mes sons et puis } \\
\text { la chaleur. Et c'est elle qui m'a } \\
\text { enseigné cette écoute comme ça, } \\
\text { parce que le chant comme ça du feu } \\
\text { sur le produit est un enseignement. " }\end{array}$ \\
\hline
\end{tabular}

AP = Alain Passard, ITV = Interview .

Figure 4 : Extraits de la partition verbale consacrant le " sonore"

\section{Conjugaison de deux sens ou plus}

Il n'est pas nécessaire de multiplier les exemples tendant à prouver qu'Alain Passard est un créateur d'inspiration lictionnelle. Les quelques fragments de la figure 5 illustrent néanmoins les reliances complexes à l'œuvre lorsqu'il associe deux sens ou plus.

\section{Détermination de l'unité thématique}

L'analyse effectuée ci-dessus nous permet, désormais, de formuler sans hésitation le thème du documentaire : il s'agit de la création d'art culinaire. Ceci justifie $a$ posteriori la catégorisation effectuée dès l'introduction, en vertu de laquelle nous avions rangé ce document dans le genre des documentaires d'art.

Le point de vue, le regard particulier porté sur ce thème et qui va compléter la détermination de l'unité thématique peut être exprimé par : la participation des cinq sens dans la créativité culinaire d'Alain Passard, ou encore : Alain Passard peintre virtuose du légume. 


\begin{tabular}{|c|c|c|c|c|c|}
\hline Chap. & TC-In & TC-Out & Durée & Rapport Image & Rapport Son \\
\hline 1 & $02: 24$ & $02: 41$ & $37 "$ & $\begin{array}{l}\text { AP au piano de } \\
\text { L'Arpège avec } \\
\text { son second }\end{array}$ & $\begin{array}{l}\text { "D'abord on a un très bel accord de } \\
\text { couleurs. Olfactivement, il y a } \\
\text { quelque chose. Gustativement, il y a } \\
\text { la douceur de la poire sur le côté un } \\
\text { petit peu arrogant de l'oignon qui est } \\
\text { intéressant. Le tout flambé donc avec } \\
\text { une petite pointe d'alcool donne un } \\
\text { caractère assez étonnant au plat. " }\end{array}$ \\
\hline 2 & $05: 40$ & $06: 08$ & $28 "$ & ITV AP & $\begin{array}{l}\text { "C'est justement cette ouverture, } \\
\text { cette créativité qui est intéressante, } \\
\text { parce que d'abord c'est un produit } \\
\text { qui appelle énormément. La } \\
\text { créativité du légume, c'est une } \\
\text { ivresse. C'est débordant. Quand on } \\
\text { s'intéresse un petit peu à cette pulpe, } \\
\text { on la dessine, on la découpe, les } \\
\text { couleurs, les parfums font que l'on } \\
\text { développe un autre sens créatif que } \\
\text { face à la cuisine animale. " }\end{array}$ \\
\hline 3 & $13: 10$ & $13: 27$ & $17 "$ & $\begin{array}{l}\text { Échange AP- } \\
\text { Sylvain Picard } \\
\text { (ses } \\
\text { interventions } \\
\text { dans le } \\
\text { dialogue sont } \\
\text { coupées) }\end{array}$ & $\begin{array}{l}\text { "Tu imagines ce côté blanc de l'ail, } \\
\text { ce jaune blé de la pomme de terre et } \\
\text { ce vert céladon de la fève, une belle } \\
\text { assiette creuse, chaude, tu vois, un } \\
\text { truc fumant, mais... tu peux faire un } \\
\text { des plus beaux plats du monde..." }\end{array}$ \\
\hline 10 & $48: 29$ & $48: 49$ & $20 "$ & AP & $\begin{array}{l}\text { "Ce qui est surtout intéressant, c'est } \\
\text { cette démarche de la cuisine par les } \\
\text { couleurs qui n'est pas réellement } \\
\text { exploitée aujourd'hui, et d'essayer de } \\
\text { donner presque un parfum et une } \\
\text { saveur à une couleur, c'est une } \\
\text { démarche qui est assez drôle. On a } \\
\text { juste, je dirais, à s'orienter sur un } \\
\text { axe. On prend un axe de couleur, on } \\
\text { choisit une couleur, et on reste sur } \\
\text { cet axe. " }\end{array}$ \\
\hline
\end{tabular}

AP = Alain Passard, ITV = Interview.

Figure 5 : Extraits de la partition verbale convoquant plus d'un sens

Ceci est confirmé par l'édition de la partition micro-structurelle du chapitre 11, qui est effectivement conforme à l'écriture de la " coda " musicale. On y voit en effet une envolée d'arpèges culinaires plus virtuoses les uns que les autres, dans des registres de gammes colorées alternant le blanc et le rose-rouge ; le tout est accompagné par un contrepoint très subtil d'arpèges musicaux et ponctué visuellement par la fermeture des portes battantes qui séparent la cuisine de la salle de L'Arpège. 


\begin{tabular}{|c|c|c|c|c|c|}
\hline $\mathrm{N}^{\circ}$ & TC-In & TC-Out & Durée & Rapport Image & Rapport Son \\
\hline 1 & $51: 04$ & $51: 06$ & $2 "$ & $\begin{array}{l}\text { Enlèvement, dos d'un serveur, } \\
\text { fermeture portes battantes } \\
\text { Plan épaule, léger recadré }\end{array}$ & $\begin{array}{l}\text { Musique : } \\
\text { envolée } \\
\text { d'arpèges au } \\
\text { piano, cordes en } \\
\text { pizzicato Son } \\
\text { d'ambiance }\end{array}$ \\
\hline 2 & $51: 06$ & $51: 11$ & $5 "$ & $\begin{array}{l}\text { Enlèvement plat d'œufs, } \\
\text { fermeture portes battantes } \\
\text { Plan épaule dans le mouvement }\end{array}$ & Idem \\
\hline 3 & $51: 11$ & $51: 17$ & $6 "$ & $\begin{array}{l}\text { Enlèvement plat blanc } 1 \text {, } \\
\text { fermeture portes battantes } \\
\text { Plan épaule dans le mouvement }\end{array}$ & Idem \\
\hline 4 & $51: 17$ & $51: 23$ & $6 "$ & $\begin{array}{l}\text { Enlèvement écrevisses, } \\
\text { fermeture portes battantes } \\
\text { Plan épaule dans le mouvement }\end{array}$ & Idem \\
\hline 5 & $51: 23$ & $51: 27$ & $4 "$ & $\begin{array}{l}\text { Enlèvement plat blanc } 2 \text {, } \\
\text { fermeture portes battantes } \\
\text { Plan épaule dans le mouvement }\end{array}$ & Idem \\
\hline 6 & $51: 27$ & $51: 35$ & $8 "$ & $\begin{array}{l}\text { Enlèvement plat rose, fermeture } \\
\text { portes battantes } \\
\text { Plan épaule dans le mouvement, } \\
\text { fondu au noir }\end{array}$ & Idem \\
\hline 7 & $51: 35$ & $51: 47$ & $12 "$ & $\begin{array}{l}\text { Légume jaune, GP } \\
\text { Lancement déroulant générique }\end{array}$ & $\begin{array}{l}\text { Musique : } \\
\text { arpèges + entrée } \\
\text { thème principal } \\
\text { au piano } \\
\text { Disparition } \\
\text { ambiance }\end{array}$ \\
\hline 8 & $51: 47$ & $51: 59$ & $12 "$ & Assiette tomates, GP & Idem \\
\hline 9 & $51: 59$ & $52: 05$ & $6 "$ & Tomates, TGP & $\begin{array}{l}\text { Musique : plus } \\
\text { d'ampleur, et de } \\
\text { volume, avec } \\
\text { violon solo et } \\
\text { cordes }\end{array}$ \\
\hline 10 & $52: 05$ & $52: 12$ & $7 "$ & Légume rouge, TTGP & Idem \\
\hline 11 & $52: 12$ & $52: 16$ & $4 "$ & $\begin{array}{l}\text { Assiette Salade Arlequin, GP } \\
\text { Fermeture fondu au noir }\end{array}$ & $\begin{array}{l}\text { Fade out sonore } \\
\text { sur un arpège } \\
\text { piano }\end{array}$ \\
\hline
\end{tabular}

$\mathrm{GP}=$ gros plan, $\mathrm{TGP}$ = trés gros plan, TTGP : très très gros plan.

Figure 6 : Partition micro-structurelle du chapitre 11 " coda " 


\section{Conclusion}

Cet article a mis en évidence l'extrême précision de l'écriture filmique convoquée dans le documentaire Alain Passard, le solfege du légume. Il a mis en œuvre une méthode de dépouillement filmique fondée sur l'édition des partitions filmiques du document, outils qui permettent une description objective du document, préalable à l'analyse approfondie de son écriture.

L'ensemble a montré que ce sont les lictions entre données qui qualifient l'audiovisuel pour représenter le gourmand. Il a en outre permis de déguster, via l'image et le son, les compositions virtuoses du grand créateur qu'est Alain Passard.

\section{SYLVIE LELEU-MERVIEL}

